\title{
Impact of cancer on adherence to glucose-lowering drug treatment in individuals with diabetes
}

\author{
Marjolein M. J. Zanders • Harm R. Haak • \\ Myrthe P. P. van Herk-Sukel • \\ Lonneke V. van de Poll-Franse • Jeffrey A. Johnson
}

Received: 9 September 2014 / Accepted: 30 December 2014 /Published online: 1 February 2015

(C) Springer-Verlag Berlin Heidelberg 2015

\begin{abstract}
Aims/hypothesis Adherence to glucose-lowering drug (GLD) treatment regimens is crucial for metabolic control and improving prognosis. Because a diagnosis of cancer might have an impact on adherence to medication, this study explored changes in adherence to GLDs following a cancer diagnosis.
\end{abstract}

Electronic supplementary material The online version of this article (doi:10.1007/s00125-015-3497-8) contains peer-reviewed but unedited supplementary material, which is available to authorised users.

M. M. J. Zanders $(\bowtie) \cdot$ L. V. van de Poll-Franse

Department of Research, Netherlands Comprehensive Cancer Organisation, P. O. Box 231, 5600 AE Eindhoven, the Netherlands e-mail: m.zanders@iknl.nl

H. R. Haak

Department of Internal Medicine, Máxima Medical Centre,

Eindhoven/Veldhoven, the Netherlands

H. R. Haak

Department of Internal Medicine, Division of General Internal Medicine, Maastricht University Medical Centre+,

Maastricht, the Netherlands

\section{H. R. Haak}

Department of Health Services Research, CAPHRI School for Public Health and Primary Care, Maastricht University,

Maastricht, the Netherlands

M. P. P. van Herk-Sukel

PHARMO Institute for Drug Outcomes Research,

Utrecht, the Netherlands

L. V. van de Poll-Franse

CoRPS-Center of Research on Psychology in Somatic Diseases,

Department of Medical and Clinical Psychology, Tilburg University,

Tilburg, the Netherlands

J. A. Johnson

School of Public Health, University of Alberta, Edmonton,

$\mathrm{AB}$, Canada
Methods All new users of GLDs between 1998 and 2011 who lived in the Eindhoven Cancer Registry-PHARMO Database Network catchment area were selected. Those with a primary cancer diagnosis during follow-up were considered cases and matched with eligible controls without cancer during followup. Medication possession ratio (MPR) was used as indicator for medication adherence. Segmented linear auto-regression analysis with interrupted time series was used to assess changes in MPR for cases compared with controls (i.e. overall trend) due to (any) cancer diagnosis and specific cancer types. Results From the 52,228 GLD users selected, 3,281 cases with cancer and 12,891 controls without cancer during follow-up were included in the study. In our analyses, before cancer diagnosis the MPR increased by $0.10 \%$ per month $(95 \%$ CI $0.10,0.10)$. Besides a significant drop in MPR at the time of cancer diagnosis of $-6.3 \%(95 \% \mathrm{CI}-6.5,-6.0)$, there was an ongoing, yet lower, monthly decline in MPR $(-0.20 \% ; 95 \%$ CI $-0.21,-0.20)$ after cancer diagnosis. The largest drops in MPR at the time of cancer diagnosis, in the range of $11-15 \%$, were seen among patients with stage IV disease and gastrointestinal or pulmonary cancers.

Conclusions/interpretation Our findings indicate a clear decline in adherence to GLD treatment regimens following a cancer diagnosis. The reason for the decline in MPR needs to be further elucidated.

Keywords Adherence $\cdot$ Cancer $\cdot$ Glucose-lowering drugs

$\begin{array}{ll}\text { Abbreviations } \\ \text { ATC } & \text { Anatomical therapeutic chemical } \\ \text { ECR } & \text { Eindhoven Cancer Registry } \\ \text { GLD } & \text { Glucose-lowering drug } \\ \text { MPR } & \text { Medication possession ratio } \\ \text { PHARMO } & \text { PHARmacoMOrbidity } \\ \text { TNM } & \text { Tumour, node, metastasis }\end{array}$




\section{Introduction}

Cancer patients with diabetes have a significantly higher overall mortality risk than those without diabetes [1-3]. To understand the association between diabetes and cancer, the American Diabetes Association and American Cancer Society reviewed the state of science regarding this in 2010 [4]. One of the key goals of the review was to gain a better understanding of whether diabetes influences cancer prognosis above and beyond the prognosis conferred by each disease independently. Since that report, most research has focussed on the influence of diabetes and glucose-lowering drugs (GLDs) on outcomes after cancer diagnosis; yet, on the contrary, cancer might affect outcomes associated with diabetes.

Achievement of normal or near normal glycaemia $\left(\mathrm{HbA}_{1 \mathrm{c}}\right.$ goal of $<7 \%$ [53 mmol $/ \mathrm{mol}]$ [5]) in individuals with diabetes is strongly linked with adherence to medication regimens [6, 7]. Overall, only $65-85 \%$ of GLD users are regarded as adherent $[8,9]$; this might decrease even more after a diagnosis of cancer. If a cancer diagnosis can influence medication adherence among GLD users, this could also affect $\mathrm{HbA}_{1 \mathrm{c}}$ levels, leading to poor metabolic control, higher risk of diabetes complications and worse overall mortality. Today, only one - very recent - study has examined the impact of a cancer diagnosis on medication adherence. Among 509 individuals with diabetes, the diagnosis of breast cancer was associated with a decline in medication adherence-measured using the medication possession ratio (MPR)-from $86 \%$ to $49 \%$ [10]. Although the investigators measured the MPR among those with breast cancer, the natural course of adherence to GLD regimens in diabetic patients without cancer is unknown. Therefore, we aimed to evaluate how a cancer diagnosis changes adherence to GLD regimens, taking into account the changes in adherence to GLDs among those without cancer.

\section{Methods}

Data sources Data were obtained from the PHARMO (PHARmacoMOrbidity) Database Network and linked at the individual patient level to the Eindhoven Cancer Registry (ECR). The catchment area covered a demographic region in the southern part of the Netherlands, and generated data for approximately one million inhabitants. The construction and validity of the ECR-PHARMO cohort have been described elsewhere [11]. The PHARMO Database Network is a large, patient-centred data network including multiple linked observational databases designed for drug safety and outcome research. For this study the community pharmacy (outpatient) database was used; this includes data on the dispensed drug, dispensing date and amount dispensed, and thus the duration of use could be calculated. All drugs are coded according to the anatomical therapeutic chemical (ATC) classification [12]. The ECR, maintained by the Netherlands Comprehensive Cancer Organisation (IKNL), records data on all patients newly diagnosed with cancer in the southern part of the Netherlands, an area with 2.4 million inhabitants. Six pathology departments, ten community hospitals and two radiotherapy departments notify the registry. Trained registration clerks actively collect data on patient characteristics, cancer diagnosis, staging and initial treatment from hospital medical records. Both the ECR and the PHARMO Database Network are recognised as high-quality sources for epidemiological research that collect information in overlapping regions in the Netherlands for a period of at least 10 years [11]. The generation of the linked dataset was approved by the responsible regulatory bodies.

Study population The source population included all patients living in the geographical region of the ECR-PHARMO cohort who were aged over 30 years and who had dispensing of GLDs (ATC code: A10) between 1 January 1998 and 31 December 2011 ( $n=81,928)$. From this source population, we selected incident users of GLDs who had two or more dispensings of GLDs preceded by a 6-month period without any GLD dispensing $(n=52,228)$.

Users of GLDs with a primary diagnosis of any cancer (except non-melanoma skin cancer) were considered cases and the date of the first cancer diagnosis (i.e. cancer confirmed by pathology) was set as the time of the event. Those GLD users without a diagnosis of cancer were eligible as controls. By including controls we were able to account for the overall course (i.e. secular trend) in medication adherence seen in individuals with diabetes. Cases and controls were matched-with replacement and a maximum of four controls per case-for age (according to 5 year age groups), sex, duration of follow-up (controls needed to have a similar or longer duration of follow-up than the total follow-up time for their cases), calendar year of first GLD dispensing (according to 2 year periods) and type of first dispensed GLD (metformin monotherapy, sulfonylurea derivative monotherapy, any insulin or other GLD groups). Both the time until the diagnosis of cancer and the total time of follow-up for the cases was then set as the same time for their controls. Because controls did not have an 'actual' cancer diagnosis, we needed to define an index date for the controls. We assigned this as the date associated with the same duration of GLD use at cancer diagnosis as for their case.

For the primary analysis we were interested in the change in medication adherence potentially associated with any cancer diagnosis, but also associated with one of the six most frequent cancer types/groups classified according to the International Classification of Diseases of Oncology (ICD-O; www.who.int/classifications/icd/adaptations/oncology/en/): colorectal (C18-20), other gastrointestinal (C15-17, C21-C26; 
oesophageal, stomach, pancreas and liver), prostate (C61), breast $(\mathrm{C} 50)$, pulmonary $(\mathrm{C} 33-34, \mathrm{C} 45)$ and urinary (C64-68) cancer.

Drug episodes for measuring medication adherence For each oral drug dispensing (i.e. except insulin: ATC code A10A [12]), the duration of use was calculated by dividing the number of tablets dispensed by the number of tablets to be used per day, as defined in the outpatient pharmacy database. For insulin, the duration of use is not often registered in the pharmacy. The intended period of use for which insulin was dispensed was set to 90 days when the duration of use was missing or considered unlikely.

All dispensings for GLDs, regardless of type, were converted into episodes of uninterrupted use. For each dispensing, the duration of use was calculated and converted into episodes of consecutive use based on the method of Catalan [13]. In this method, the time span was the date of the first dispensing until the end date of the final dispensing together with the permissible gap. This gap was determined to be either half the period of the given dispensing or 7 days, whichever was greater. Because many patients resumed the same treatment within 2 months of the end of the previous episode, we expanded the permissible gap between drug dispensings of the same drug with an additional 45 days.

We used the MPR as an indicator for medication adherence, representing the amount of medication patients had in possession over a certain time period. Thus, a $10 \%$ decline in MPR translates to a difference of 3 days in a 30-day month that is not covered by the use of GLDs due to the cancer diagnosis. The MPR was calculated every month for both cases and controls by dividing the cumulative days of drug exposure by the total number of days in that time window [14]. Last, for every month (i.e. every time window) the MPR for cases was compared with the MPR for matched controls; these controls represented the overall trend among individuals with diabetes but without cancer. Thus, the impact of cancer on MPR for cases was set against this background trend.

Statistical analyses Differences in demographic and clinical characteristics between users of GLDs with any cancer and their controls were analysed using $\chi^{2}$ and $t$ tests where applicable.

We used the method for interrupted time-series analysis [15], with monthly time-windows for the MPR in cases and controls. A segmented linear auto-regression analysis was used to statistically measure the changes in MPR in intercept and slope in the post-cancer period compared with the precancer period. The regression model used to fit our data included a continuous variable for time from first dispensing of GLDs until the end of that time window, a binary variable for time occurring before or after the diagnosis of cancer and a continuous variable for time after cancer. The parameter estimates for the binary variable as well as for the variable for the time after cancer are of main interest, whereas the parameter for time from first dispensing of GLDs controls for the overall trend in MPR regardless of a cancer diagnosis. We calculated the Durbin-Watson statistic to test for the serial autocorrelation of the error terms in the regression models and corrected for any autocorrelation according to the order (number of lags) which was given by the Durbin-Watson statistic to be significant. All final models had a Durbin-Watson statistic value close to the preferred value of 2 [16]. A $p$ value of $<0.05$ was considered statistically significant. Analyses were performed using SAS software (version 9.4; SAS institute, Cary, IN, USA).

Regression analyses were performed for all cancers combined and for the previously mentioned cancer types separately. In addition, we stratified the analyses for the tumour, node, metastasis (TNM) stage of cancer as well as for the cancer treatment received to explore their effects on the medication adherence in our study population. Additional subgroup analyses were performed, in which we stratified according to age group ( $<60$ years, $\geq 60$ and $<70$ years, $\geq 70$ years) and according to the type of GLD used at cancer diagnosis, because a cancer diagnosis might affect adherence differently in these subgroups.

Because patients recently started on GLDs might differ in their medication adherence compared with long-time users, those who started using GLDs in the 6 months prior to cancer diagnosis were excluded in a sensitivity analyses. To evaluate whether medication adherence was influenced directly by mortality, a sensitivity analysis was performed in which only those patients who died during follow-up were included.

\section{Results}

From the ECR-PHARMO cohort, 3,281 cases with cancer and 12,891 controls without cancer were selected (Table 1). The mean (SD) age at the start of GLD use was 67.7 (9.8) years for cases and $67.5(9.7)$ years for controls $(p=0.3)$. Most patients started using GLDs before 2005. The time between the start of GLD use and the date of cancer diagnosis (or index date for controls) was 3.7 (3.0) years for both groups and the total duration of follow-up was 6.6 (3.5) years for cases and 6.5 (3.5) years for controls (Table 1). Before cancer diagnosis (or index date for the controls), $33 \%$ of the cases stopped using GLDs and among the controls the number of patients that used insulin or combination treatment increased (Table 1).

In our analyses, before cancer diagnosis the MPR increased by $0.10 \%$ per month $(95 \%$ CI $0.10,0.10$; Table 2 , Fig. 1$)$. Besides a significant drop in MPR at the time of cancer diagnosis of $-6.3 \%$ ( $95 \% \mathrm{CI}-6.5,-6.0)$, there was an ongoing yet lower monthly decline in MPR $(-0.20 \%$; $95 \%$ CI -0.21 , 
Table 1 Characteristics of study population $(N=16,172)$
Data are presented as $n(\%)$ unless specified otherwise

Index date: because controls did not have an 'actual' cancer diagnosis, we needed to define an index date for the controls. We assigned this as the date associated with the same duration of GLD use at cancer diagnosis as for their case n.a., not applicable

\begin{tabular}{|c|c|c|c|}
\hline Characteristic & $\begin{array}{l}\text { Users of GLDs who } \\
\text { developed cancer } \\
(n=3,281)\end{array}$ & $\begin{array}{l}\text { Matched users } \\
\text { of GLDs without } \\
\text { cancer }(n=12,891)\end{array}$ & $p$ value \\
\hline Age at first GLD dispensing, years, mean (SD) & $67.7(9.8)$ & $67.5(9.7)$ & 0.3 \\
\hline Male sex & $1,839(56)$ & $7,218(56)$ & 1.0 \\
\hline \multicolumn{4}{|l|}{ Year of initiation of GLDs use } \\
\hline 1998-2001 & $1,030(32)$ & $4,030(31)$ & \\
\hline $2002-2005$ & $1,286(39)$ & $5,059(39)$ & \\
\hline 2006-2009 & $860(26)$ & $3,386(27)$ & \\
\hline $2010-2011$ & $105(13)$ & $416(3)$ & 1.0 \\
\hline \multicolumn{4}{|l|}{ Use of GLDs } \\
\hline \multicolumn{4}{|l|}{ At start of use of GLDs } \\
\hline Metformin monotherapy & $1,627(50)$ & $6,442(50)$ & \\
\hline Sulfonylurea derivatives monotherapy & $1,308(40)$ & $5,163(40)$ & \\
\hline Insulin (monotherapy or combination with) & $199(6)$ & $742(6)$ & \\
\hline Other GLDs & $147(4)$ & $544(4)$ & 0.8 \\
\hline \multicolumn{4}{|l|}{ At cancer diagnosis/index date } \\
\hline Metformin monotherapy & $836(25)$ & $4,144(32)$ & \\
\hline Sulfonylurea derivatives monotherapy & $826(25)$ & $3,434(27)$ & \\
\hline $\begin{array}{l}\text { Combination of metformin and } \\
\text { sulfonylurea derivatives }\end{array}$ & $155(5)$ & $1,032(8)$ & \\
\hline Insulin (monotherapy or combination with) & $280(9)$ & $1,438(11)$ & \\
\hline Other GLDs & $112(3)$ & $620(5)$ & \\
\hline No use of GLDs & $1,072(33)$ & $2,223(17)$ & $<0.0001$ \\
\hline \multicolumn{4}{|l|}{ Cancer type } \\
\hline Colorectal cancer & $549(17)$ & n.a. & \\
\hline Oesophageal, stomach, pancreas or liver cancer & $387(12)$ & & \\
\hline Prostate cancer & $377(11)$ & & \\
\hline Breast cancer & $415(13)$ & & \\
\hline Pulmonary cancers & $425(13)$ & & \\
\hline Urinary cancer & $390(12)$ & & \\
\hline Other types of cancer & $738(22)$ & & \\
\hline $\begin{array}{l}\text { Time between start of GLD treatment and } \\
\text { cancer/index date, years, mean (SD) }\end{array}$ & $3.7(3.0)$ & $3.7(3.0)$ & 0.8 \\
\hline \multicolumn{4}{|l|}{ TNM stage } \\
\hline Non-invasive & $249(8)$ & n.a. & \\
\hline I & $679(21)$ & & \\
\hline II & $972(20)$ & & \\
\hline III & $498(15)$ & & \\
\hline IV & $612(19)$ & & \\
\hline Unknown & $571(17)$ & & \\
\hline \multicolumn{4}{|l|}{ Received cancer treatment } \\
\hline Surgery & $1,725(53)$ & n.a. & \\
\hline Chemotherapy & $719(22)$ & & \\
\hline Radiotherapy & $827(25)$ & & \\
\hline \multicolumn{4}{|l|}{ Follow-up } \\
\hline Duration of follow-up (years; means (SD)) & $6.6(3.5)$ & $6.5(3.5)$ & 0.5 \\
\hline \multicolumn{4}{|l|}{ End of follow-up } \\
\hline Death & $1,189(36)$ & n.a. & \\
\hline Lost to follow-up & $42(1)$ & & \\
\hline Reached end of follow-up & $2,050(63)$ & & \\
\hline
\end{tabular}


Table 2 Medication adherence assessed by change in MPR (\%) during follow-up due to the diagnosis of cancer

\begin{tabular}{|c|c|c|c|c|c|c|c|c|c|}
\hline \multirow[t]{2}{*}{ Cancer diagnosis } & \multirow[t]{2}{*}{$n_{\text {cases }} / n_{\text {controls }}$} & \multicolumn{2}{|c|}{$\begin{array}{l}\text { Intercept at first drug } \\
\text { dispensing }\end{array}$} & \multicolumn{2}{|c|}{$\begin{array}{l}\text { Time before cancer/ } \\
\text { index date (per month) }\end{array}$} & \multicolumn{2}{|c|}{$\begin{array}{l}\text { Intercept at cancer } \\
\text { diagnosis }\end{array}$} & \multicolumn{2}{|c|}{$\begin{array}{l}\text { Time after cancer diagnosis } \\
\text { (per month) }\end{array}$} \\
\hline & & $\operatorname{MPR} \Delta$ & $95 \% \mathrm{CI}$ & MPR $\Delta$ & $95 \% \mathrm{CI}$ & $\operatorname{MPR} \Delta$ & $95 \% \mathrm{CI}$ & $\operatorname{MPR} \Delta$ & $95 \% \mathrm{CI}$ \\
\hline Any cancer & $3,281 / 12,891$ & 0.4 & $0.1,0.8^{*}$ & 0.10 & $0.10,0.10^{* * * *}$ & -6.3 & $-6.5,-6.0 * *$ & -0.20 & $-0.21,-0.20^{* * * *}$ \\
\hline \multicolumn{10}{|c|}{ Stratified for type of cancer } \\
\hline Colorectal cancer & $549 / 2,154$ & 1.0 & $0.1,1.9^{*}$ & 0.13 & $0.12,0.14 * * * *$ & -8.3 & $-9.0,-7.7 * * * *$ & -0.17 & $-0.19,-0.16^{* * * *}$ \\
\hline $\begin{array}{l}\text { Oesophageal, } \\
\text { stomach, pancreas } \\
\text { or liver cancer }\end{array}$ & $387 / 1,526$ & -0.1 & $-1.3,1.0$ & 0.10 & $0.09,0.11^{* * * *}$ & -12.5 & $-13.4,-11.6^{* * * *}$ & -0.45 & $-0.47,-0.42 * * * *$ \\
\hline Prostate cancer & $377 / 1,463$ & 1.8 & $0.8,2.9^{*}$ & 0.02 & $0.01,0.03^{*}$ & 2.1 & $1.4,2.8 * * * *$ & -0.07 & $-0.09,-0.05 * * * *$ \\
\hline Breast cancer & $415 / 1,617$ & 1.7 & $0.7,2.8^{*}$ & 0.03 & $0.02,0.04 * * * *$ & -0.5 & $-1.2,0.3$ & -0.09 & $-0.10,-0.07 * * * *$ \\
\hline Pulmonary cancers & $425 / 1,694$ & -0.3 & $-1.2,0.7$ & 0.15 & $0.14,0.16^{* * * *}$ & -15.2 & $-16.0,-14.4 * *$ & -0.54 & $-0.56,-0.52 * * * *$ \\
\hline Urinary cancer & $390 / 1,528$ & -3.1 & $-4.2,-2.0 * * * *$ & 0.17 & $0.15,0.18^{* * * *}$ & -0.8 & $-1.5,-0.1 *$ & -0.38 & $-0.40,-0.36^{* * * *}$ \\
\hline
\end{tabular}

Index date: because controls did not have an 'actual' cancer diagnosis, we needed to define an index date for the controls. We assigned this as the date associated with the same duration of GLDs use at cancer diagnosis as for their case

${ }^{*} p<0.05$, comparing cancer cases with their controls; $* * * * p<0.0001$, comparing cancer cases with their controls

MPR $\Delta$, absolute change in medication possession ratio (\%)

-0.20) after cancer diagnosis, both indicating a clear decline in medication adherence because of cancer.

When we stratified the analysis for the type of cancer, different effects were seen for the various tumour types (Table 2, Fig. 2). While no important decline in MPR was seen at the time of diagnosis for prostate cancer $(2.1 \% ; 95 \%$ CI $1.4,2.8)$ or breast cancer $(-0.5 \% ; 95 \% \mathrm{CI}-1.2,0.3)$, large drops were seen in patients with oesophageal, stomach, pancreas or liver cancers $(-12.5 \%$; $95 \% \mathrm{CI}-13.4,-11.6)$ and pulmonary cancers $(-15.2 \%$; $95 \%$ CI $-16.0,-14.4)$ (Fig. 2). Among those patients with large drops, the MPR after cancer diagnosis

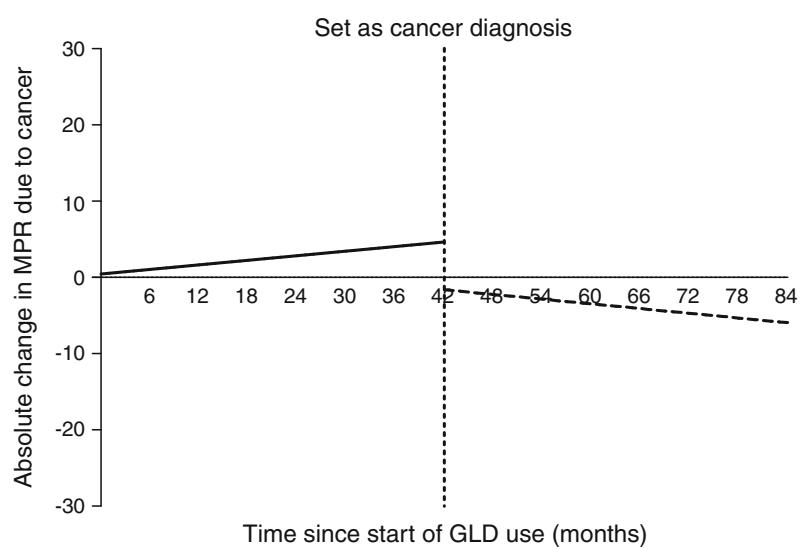

Fig. 1 Absolute change in MPR due to cancer, in which the diagnosis of any cancer was set at 42 months ( 3.5 years). The change in MPR for cases was set against the background trend (i.e. the overall trend in MPR among individuals with diabetes/the controls). The solid line represents the change in MPR (\%) before cancer diagnosis and the dashed line represents the change in MPR (\%) after cancer diagnosis decreased by approximately $0.5 \%$ each month, indicating ongoing declining medication adherence in cases (oesophageal, stomach, pancreas or liver cancers $-0.45 \% ; 95 \% \mathrm{CI}-0.47$, -0.42 ; pulmonary cancers $-0.54 \% ; 95 \% \mathrm{CI}-0.56,-0.52$ ). In patients with oesophageal, stomach, pancreas or liver cancers, the largest declines were seen for liver and oesophageal cancers $(-35.3 \%$; $95 \% \mathrm{CI}-39.1,-31.5$ and $-19.2 \%$; $95 \% \mathrm{CI}$ $-21.0,17.4$; respectively; electronic supplementary material [ESM] Table 1). Within the group of pulmonary cancers, small cell and non-small cell lung cancer had comparable declines in MPR (ESM Table 1). However, for each extra month after cancer diagnosis, the MPR declined further, with almost $1 \%$ change each month for pancreas and small cell lung cancers $(-0.97 \% ; 95 \% \mathrm{CI}-1.01,-0.93$ and $-0.89 \%$; $95 \%$ CI $-0.95,-0.84$, respectively; ESM Table 1). When comparing patients with colon cancer and rectal cancer, the degree of the drop in MPR at cancer diagnosis remained for colon cancer patients, whereas it disappeared for rectal cancer patients (ESM Table 1).

The higher the TNM stage, the greater the observed decline in medication adherence at cancer diagnosis (Table 3). For patients with stage IV disease, the drop in MPR was $-10.7 \%$ $(95 \%$ CI $-11.3,-10.1 \%)$, while in each extra month after cancer diagnosis the MPR declined an additional $-0.64 \%$ (95\% CI $-0.66,-0.62)$. Although no effect modification by chemotherapy or radiotherapy administration was seen, cancer patients who did not receive surgery had a more pronounced drop in MPR at cancer diagnosis $(-10.8 \%$; $95 \% \mathrm{CI}$ $-11.2,-10.4)$ than those who did receive surgery $(-2.8 \%$; $95 \%$ CI $-3.1,-2.4$; Table 3). The impact of cancer on 
a

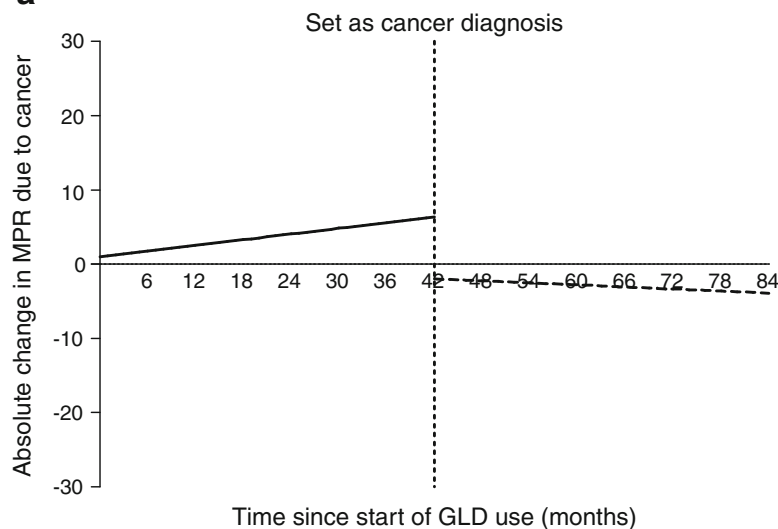

C

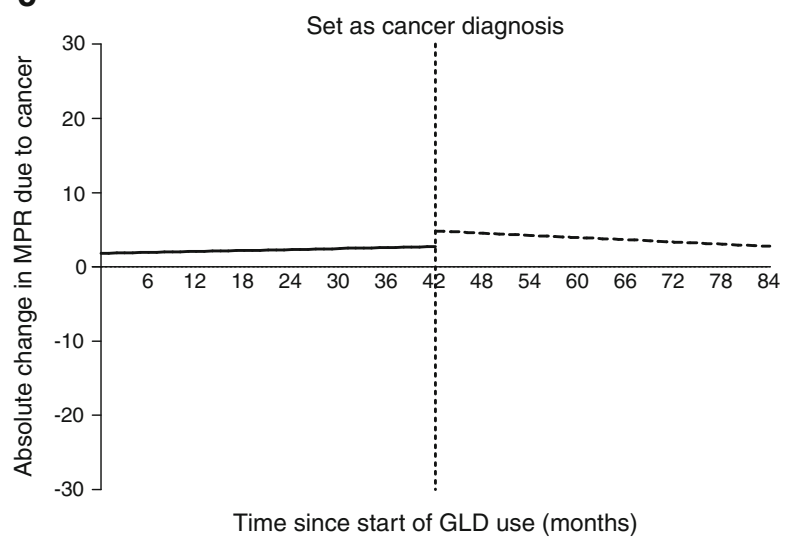

e

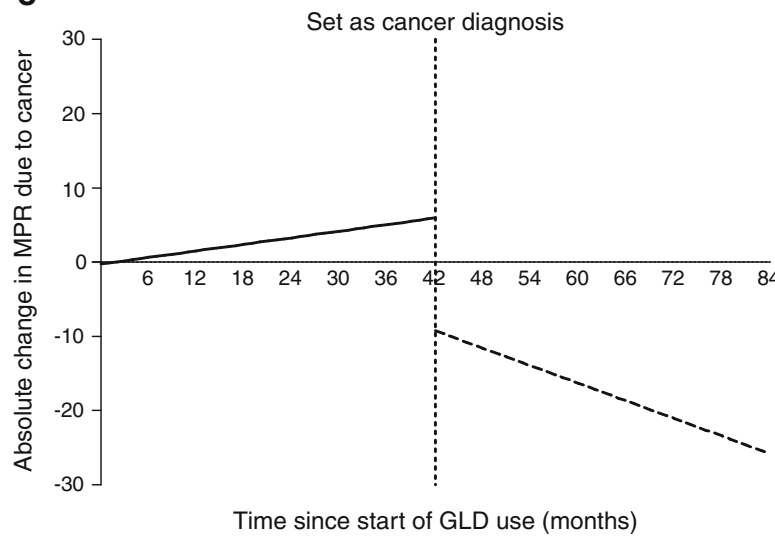

Fig. 2 Absolute change in MPR due to different cancer types, in which the diagnosis of cancer was set at 42 months ( 3.5 years). The change in MPR for cases was set against the background trend (i.e. the overall trend in MPR among individuals with diabetes/the controls). The solid line represents the change in MPR (\%) before cancer diagnosis and the dashed

medication adherence was significant for all age groups, but with larger decreases in MPR observed with increasing age. Moreover, after cancer diagnosis, the decline in MPR was larger with increasing age (Table 3). The impact of a cancer diagnosis on MPR was most apparent among patients who used sulfonylurea derivatives in combination with metformin b

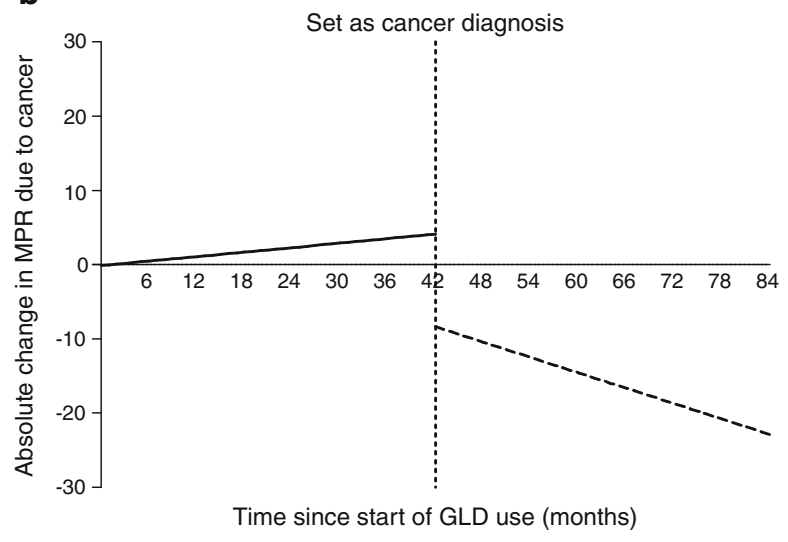

d
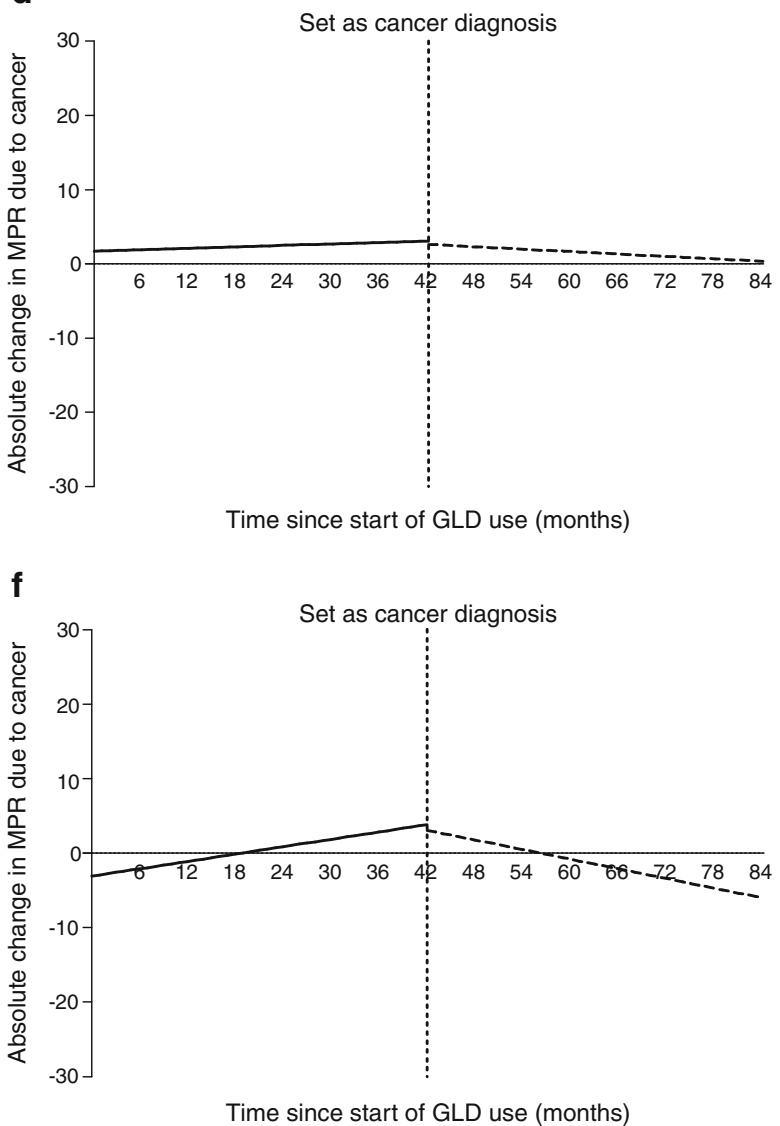

line represents the change in MPR (\%) after cancer diagnosis. The changes in MPR due to colorectal cancer (a), oesophageal, stomach, pancreas or liver cancer (b), prostate cancer (c), breast cancer (d), pulmonary cancers (e) and urinary cancer $(\mathbf{f})$ are shown

and among patients who used insulin as monotherapy or combination therapy at cancer diagnosis (Table 3). The inclusion of only long-time GLDs users or the inclusion of those who died during follow-up resulted in comparable estimates for the MPR at cancer diagnosis and for the monthly change in MPR after cancer diagnosis (Table 3). 
Table 3 Subgroup analyses of medication adherence assessed by change in MPR (\%) during follow-up due to the diagnosis of cancer

\begin{tabular}{|c|c|c|c|c|c|c|c|c|c|}
\hline \multirow[t]{2}{*}{ Characteristic } & \multirow[t]{2}{*}{$n_{\text {cases }} / n_{\text {controls }}$} & \multicolumn{2}{|c|}{$\begin{array}{l}\text { Intercept at first drug } \\
\text { dispensing }\end{array}$} & \multicolumn{2}{|c|}{$\begin{array}{l}\text { Time before cancer/index } \\
\text { date (per month) }\end{array}$} & \multicolumn{2}{|c|}{ Intercept at cancer diagnosis } & \multicolumn{2}{|c|}{$\begin{array}{l}\text { Time after cancer } \\
\text { diagnosis (per month) }\end{array}$} \\
\hline & & MPR $\Delta$ & $95 \% \mathrm{CI}$ & MPR $\Delta$ & $95 \% \mathrm{CI}$ & MPR $\Delta$ & $95 \% \mathrm{CI}$ & $\operatorname{MPR} \Delta$ & $95 \% \mathrm{CI}$ \\
\hline \multicolumn{10}{|l|}{ TNM stage } \\
\hline I & $679 / 2,677$ & -0.5 & $-1.6,0.6$ & 0.07 & $0.07,0.08 * * * *$ & 0.3 & $-0.2,0.8$ & -0.10 & $-0.11,-0.08 * * * *$ \\
\hline II & $972 / 2,610$ & 1.7 & $0.6,2.7^{* * * *}$ & 0.08 & $0.07,0.09^{* * * *}$ & -3.2 & $-3.7,-2.7^{* * * *}$ & -0.09 & $-0.11,-0.08 * * * *$ \\
\hline III & $498 / 1,939$ & -2.2 & $-3.4,-0.9^{* * * *}$ & 0.16 & $0.15,0.17 * * * *$ & -5.8 & $-6.4,-5.2 * * * *$ & -0.32 & $-0.34,-0.31 * * * *$ \\
\hline IV & $612 / 2,439$ & -0.6 & $-1.8,0.5$ & 0.15 & $0.14,0.16^{* * * *}$ & -10.7 & $-11.3,-10.1^{* * * *}$ & -0.64 & $-0.66,-0.62 * * * *$ \\
\hline \multicolumn{10}{|l|}{ Initial cancer treatment } \\
\hline Surgery & $1,725 / 6,760$ & 0.0 & $-0.5,0.6$ & 0.09 & $0.08,0.09 * * * *$ & -2.8 & $-3.1,-2.4 * * * *$ & -0.16 & $-0.17,-0.16^{* * * *}$ \\
\hline No surgery & $1,556 / 6,131$ & 0.7 & $0.2,1.3^{* * * *}$ & 0.11 & $0.11,0.12 * * * *$ & -10.8 & $-11.2,-10.4^{* * * *}$ & -0.29 & $-0.30,-0.28 * * * *$ \\
\hline Chemotherapy & $719 / 2,848$ & -0.1 & $-0.9,0.7$ & 0.11 & $0.10,0.12 * * * *$ & -5.2 & $-5.7,-4.6^{* * * *}$ & -0.39 & $-0.40,-0.37 * * * *$ \\
\hline No chemotherapy & $2,562 / 10,043$ & 0.6 & $0.2,1.0^{* * * *}$ & 0.10 & $0.09,0.10^{* * * *}$ & -6.4 & $-6.7,-6.1 * * * *$ & -0.17 & $-0.18,-0.16^{* * * *}$ \\
\hline Radiotherapy & $827 / 3,230$ & 0.4 & $-0.3,1.2$ & 0.07 & $0.07,0.08 * * * *$ & -3.8 & $-4.3,-3.3^{* * * *}$ & -0.15 & $-0.16,-0.13 * * * *$ \\
\hline No radiotherapy & $2,454 / 9,661$ & 0.4 & $-0.1,0.9$ & 0.11 & $0.10,0.11 * * * *$ & -7.2 & $-7.5,-6.9^{* * * *}$ & -0.23 & $-0.24,-0.22 * * * *$ \\
\hline \multicolumn{10}{|l|}{ Age at start of GLD use } \\
\hline$<60$ years & $654 / 2,573$ & 0.6 & $-0.1,1.2$ & 0.01 & $0.00,0.02 *$ & -4.2 & $-4.8,-3.7 * * * *$ & -0.16 & $-0.18,-0.15^{* * * *}$ \\
\hline$\geq 60$ and $<70$ years & $1,163 / 4,611$ & 0.5 & $0.1,1.0^{*}$ & 0.12 & $0.11,0.12 * * * *$ & -6.3 & $-6.8,-5.9^{* * * *}$ & -0.21 & $-0.22,-0.20 * * * *$ \\
\hline$\geq 70$ years & $1,464 / 5,707$ & 0.6 & $0.1,1.1 *$ & 0.17 & $0.16,0.17 * * * *$ & -8.3 & $-8.7,-7.8^{* * * *}$ & -0.27 & $-0.28,-0.26^{* * * *}$ \\
\hline \multicolumn{10}{|c|}{ Type of GLD used at cancer diagnosis ${ }^{\mathrm{a}}$} \\
\hline Monotherapy metformin & $836 / 3,304$ & -0.1 & $-0.7,0.5$ & 0.27 & $0.27,0.28 * * * *$ & -2.4 & $-2.9,-1.9 * * * *$ & -0.72 & $-0.73,-0.70 * * * *$ \\
\hline $\begin{array}{l}\text { Monotherapy } \\
\text { sulfonylurea derivative }\end{array}$ & $826 / 3,258$ & 2.7 & $2.2,3.3 * * * *$ & 0.26 & $0.25,0.27 * * * *$ & -8.8 & $-9.3,-8.3^{* * * *}$ & -0.49 & $-0.50,-0.47 * * * *$ \\
\hline Insulin & $280 / 1,100$ & 2.1 & $1.3,3.0 * * * *$ & 0.24 & $0.23,0.25 * * * *$ & -6.1 & $-7.1,-5.2 * * * *$ & -0.76 & $-0.79,-0.73 * * * *$ \\
\hline $\begin{array}{l}\text { Metformin and } \\
\text { sulfonylurea derivative }\end{array}$ & $155 / 616$ & 5.5 & $4.3,6.7^{* * * *}$ & 0.16 & $0.15,0.18^{* * * *}$ & -19.5 & $-20.7,-18.3^{* * * *}$ & -0.62 & $-0.65,-0.58^{* * * *}$ \\
\hline Other GLDs & $112 / 445$ & 1.4 & $-0.1,2.8$ & 0.12 & $0.10,0.14 * * * *$ & -2.6 & $-4.1,-1.1^{*}$ & -0.54 & $-0.58,-0.50 * * * *$ \\
\hline No use of GLDs & $1,072 / 4,168$ & -1.5 & $-2.1,-1.0^{* * * *}$ & -0.15 & $-0.16,-0.15^{* * * *}$ & -3.0 & $-3.5,-2.6^{* * * *}$ & 0.26 & $0.25,0.27 * * * *$ \\
\hline \multicolumn{10}{|l|}{ Sensitivity analyses } \\
\hline $\begin{array}{l}\text { Exclusion of users of } \\
\text { GLDs }<6 \text { months }\end{array}$ & $2,857 / 11,215$ & -0.2 & $-0.7,0.3$ & 0.11 & $0.11,0.12 * * * *$ & -5.1 & $-5.4,-4.9 * * * *$ & -0.24 & $-0.25,-0.23 * * * *$ \\
\hline $\begin{array}{l}\text { GLD users who died } \\
\text { during follow-up }\end{array}$ & $1,189 / 4,739$ & -1.7 & $-2.6,-0.9 * * * *$ & 0.23 & $0.22,0.24 * * * *$ & -5.3 & $-5.8,-4.9 * * * *$ & -0.34 & $-0.35,-0.32 * * * *$ \\
\hline
\end{tabular}

Index date: because controls did not have an 'actual' cancer diagnosis, we needed to define an index date for the controls. We assigned this as the date associated with the same duration of GLD use at cancer diagnosis as for their case

${ }^{a}$ We performed subgroup analyses in which the type of GLD used at cancer diagnosis of the case determined the subgroup

${ }^{\mathrm{b}}$ In this sensitivity analysis GLD users who started their used within the 6 months prior to cancer diagnosis were excluded

${ }^{\mathrm{c}}$ In this sensitivity analysis, only GLD users with a cancer diagnosis during follow-up who died during follow-up and their controls were included ${ }^{*} p<0.05$, comparing cancer cases with their controls; ${ }^{* * * *} p<0.0001$, comparing cancer cases with their controls

MPR $\Delta$, absolute change in medication possession ratio (\%)

\section{Discussion}

This population-based study revealed that in new GLD users, the diagnosis of cancer negatively influenced adherence to the medication regimen, with a decrease in MPR of $6 \%$ at the time of cancer diagnosis. Importantly, the influence of cancer on adherence to GLD treatment seemed to be influenced by the type of cancer, with more pronounced effects observed in patients with oesophageal, stomach, pancreas or liver cancers and pulmonary cancers. Also, more advanced cancer stages at diagnosis resulted in substantially lower MPRs at the time of cancer diagnosis.

In this study, the MPR drop of $6 \%$ at the time of any cancer diagnosis translates to a difference of 2 days in a month that is not covered by the use of GLDs due to the diagnosis of any cancer. The sensitivity of the MPR as an indicator for medication adherence has been assessed in many studies. In general, an MPR of over $80 \%$ is indicative of adherence to a drug regimen $[14,17]$. Consequently, a 20\% MPR drop because of cancer would be considered the cut-off for an adherent vs 
non-adherent GLD user. Based on these values, it may be that the overall decline of $6 \%$ we observed may not be considered clinically important. On the other hand, the decline in MPR observed in patients with more severe or advanced cancers may be considered clinically important.

Interestingly, previous studies showed that a relatively small change in MPR is associated with changes in metabolic control $[6,7]$. In one study, a statistically significant $48 \%$ decrease in the odds of poor glycaemic control $\left(\mathrm{HbA}_{1 \mathrm{c}}>8 \%\right.$ $(64 \mathrm{mmol} / \mathrm{mol}))$ was found for each percentage increase in MPR (OR 0.5; 95\% CI 0.4, 0.6) [6]. Another study showed that individuals with diabetes and recent $\mathrm{HbA}_{1 \mathrm{c}}$ values $>9.0 \%$ $(75 \mathrm{mmol} / \mathrm{mol}$; OR $1.5 ; 95 \%$ CI $1.3,1.7)$ had higher mortality than those with recent 'normal' $\mathrm{HbA}_{1 \mathrm{c}}$ values between $6.5 \%$ (48 $\mathrm{mmol} / \mathrm{mol})$ and $9 \%(75 \mathrm{mmol} / \mathrm{mol})$ [18]. Thus, the drop in MPR observed among patients with cancer in our study might have negatively influenced survival via the aforementioned mechanisms and may (partly) explain the established association between diabetes, cancer and survival [1-3].

A diagnosis of prostate cancer or breast cancer seemed to have no influence on medication adherence in GLD users, contrary to observations made in 509 US breast cancer patients [10]. In those patients, 1 year before the cancer diagnosis the MPR was $85 \%$, while during the period of treatment for cancer (cancer diagnosis until 210 days after) the MPR fell to $49 \%$ [10]. Although this decline in MPR is remarkable, the absence of a control group without breast cancer and lack of information on treatment duration are limitations of this study [10]. The control group is an important consideration because generally the MPR does tend to decrease over time among users of GLDs. In one study, in which most patients had drug treatment durations of $<10$ years, approximately two-thirds of the patients had an MPR under 65\% [19].

In our study, oesophageal, stomach, pancreas or liver cancers and pulmonary cancers had a large impact on adherence to GLD treatment regimens. While it is indisputable that falls of around $15 \%$ in MPR have their influence on metabolic control and mortality, we need to understand how medication adherence among users of GLDs was particularly influenced by these types of cancer. Compared with the other types of cancer under study, these cancer types are associated with the worst prognosis and with the lowest rates of tumour resections [20]. The hypothesis that the prognosis of cancer is associated with medication adherence is strengthened by our findings that the TNM stage [21], which is the most important prognostic factor in cancer patients, also seems to be associated with medication adherence. Evidence suggests that medication adherence in users of GLDs seems to decrease following major life events or when people are under stress [9, 22]. A diagnosis of stage IV disease could be considered such a major life event. Those users of GLDs who have more lethal cancers might prioritise the fight against cancer over the effort required to have a good metabolic control for their diabetes.
This 'life chaos' due to another disease has been investigated in post-myocardial-infarction patients [23]. After adjusting for other potential factors associated with medication non-adherence, life chaos, assessed according to responses to questions on whether they had a stable or organised life, was significantly associated with non-adherence to drugs for cardiovascular disease [23]. Last, the prognosis seemed to only partly explain the impact of cancer on medication adherence because in oesophageal cancer patients (3-year survival of $17 \%$ [20]) the diagnosis of cancer had a stronger impact on medication adherence than in patients with the most lethal form of cancer, pancreas cancer (3-year survival of 6\% [20]). This difference may also be explained by symptoms of cancer that might result in intolerable intake of oral drugs, leading to discontinuation of GLD therapy. In addition, the administration of chemotherapy might be expected to influence medication adherence, although this was not found in our subgroup analyses of patients who did or did not receive chemotherapy. In this study, the impact of a cancer diagnosis on adherence seemed to depend on the age at first use of GLDs and on the type of GLD used at cancer diagnosis. Although we matched our cases and controls on these criteria, the age and the type of GLD used (i.e. the complexity of the treatment scheme) remain important factors to consider when assessing medication adherence.

In patients with liver cancer $(n=23)$, the diagnosis of cancer strongly influenced the MPR with a $35 \%$ decline at cancer diagnosis. The association between diabetes and liver cancer might reflect some degree of 'reverse causality', with liver cancer itself or its related liver diseases (such as cirrhosis) leading to the onset of diabetes [24]. Once the tumour is removed, the insulin resistance might resolve, and the physician might advise the patient to stop using the GLDs, causing the MPR to decline. Moreover, metformin might be stopped because it is contraindicated in patients with advanced liver diseases with associated cirrhosis, ascites, or encephalopathy [25]. Although the real risk is minimal [26], the potential of metformin to cause lactic acidosis might lead physicians to withhold metformin in patients with advanced liver disease [25].

This study had many strengths, such as the inclusion of only 'new' GLD users, with a known duration of GLD use, and the inclusion of a matched control group without cancer. However, pharmacy records provide no guarantee as to whether patients were compliant with their medication prescriptions.

The MPR might be a good indicator for medication adherence, although the physician could have advised the patient to stop the treatment with GLDs, which could not be investigated. With our data, the reasons for stopping treatment for diabetes were unknown-possibly because of frequent hypoglycaemic events due to cancer [27] or intolerable oral intake of drugs. Due to the lack of longitudinal $\mathrm{HbA}_{1 \mathrm{c}}$ data, we were not able to understand whether this might be 
explained by an improvement in metabolic control; however, our previous research (Zanders, unpublished data) showed that $\mathrm{HbA}_{1 \mathrm{c}}$ values improved around the diagnosis of cancer. For this study we only had information from outpatient pharmacies that did not include data on drugs used within the hospital or within nursing homes. This might have resulted in an overestimation of MPR decline. Another limitation was that for some patients we missed information on the duration for which insulin was dispensed and we might have wrongly estimated these dispensings on 90 days.

The interrupted time series analysis is only valid to the extent that the cancer diagnosis was the only event that changed over time and the only event that was able to change the monthly calculated MPR [15]. In this study, a visit by a patient to the endocrinologist because of vague complaints of an undiagnosed cancer might be a competing event [28]. Moreover, missing dispensing data (e.g. because of hospitalisations) might falsely give the impression that medication adherence had fallen when in fact the data was simply missing. Because the PHARMO database was linked to the ECR for the cancer incidence years 1998-2011, we were not able to exclude the users of GLDs with a previous or recent cancer diagnosis. Thus, at the start of use of GLDs the medication adherence might already have been influenced by cancer.

In summary, this study revealed that the medication adherence among users of GLDs was influenced by cancer diagnosis. Although the impact of cancer was more pronounced among cancers with a worse prognosis and among those with more advanced TNM stages, the difference in prognosis associated with these cancers seemed to only partly explain the impact of cancer on medication adherence. The decline in adherence seen among users of GLDs with cancer might negatively affect survival and (partly) explain the established association between diabetes, cancer and survival [1-3]. In future studies, the reason for the decline in MPR needs to be further elucidated among the different cancer types - is it the patient who prioritises the fight against cancer or the advice of the physician to stop the treatment?

Acknowledgements The authors thank the registration team of the Netherlands Comprehensive Cancer Organisation, Eindhoven, and also the PHARMO Institute for drug outcomes research, for their dedicated data collection. We thank M. Lin of the School of Public Health, University of Alberta, Canada, for assisting with the statistical analyses performed for this manuscript.

Funding The present research was supported by a grant from the European Foundation for the Study of Diabetes (EFSD).

Duality of interest MPPH is an employee of the PHARMO Database Network for Drug Outcomes Research. This independent research institute performs financially supported studies for government and related healthcare authorities and several pharmaceutical companies. This study, however, is not financially supported by a pharmaceutical company. All other authors declare that there is no duality of interest associated with their contribution to this manuscript.
Contribution statement MMJZ contributed to the conception and design of the study, acquired and analysed the data and drafted the manuscript. MPPH, HRH, LVP and JAJ contributed to the conception and design of the study and critically reviewed the manuscript. MMJZ is the guarantor of this work. All authors approved the final version of the manuscript.

\section{References}

1. Barone BB, Yeh HC, Snyder CF et al (2008) Long-term all-cause mortality in cancer patients with preexisting diabetes mellitus: a systematic review and meta-analysis. JAMA 300:2754-2764

2. Renehan AG, Yeh HC, Johnson JA, Wild SH, Gale EA, Moller H (2012) Diabetes and cancer (2): evaluating the impact of diabetes on mortality in patients with cancer. Diabetologia 55:1619-1632

3. van de Poll-Franse LV, Houterman S, Janssen-Heijnen ML, Dercksen MW, Coebergh JW, Haak HR (2007) Less aggressive treatment and worse overall survival in cancer patients with diabetes: a large population based analysis. Int J Cancer 120:1986-1992

4. Giovannucci E, Harlan DM, Archer MC et al (2010) Diabetes and cancer: a consensus report. CA Cancer J Clin 60:207-221

5. American Diabetes Association (2014) Standards of medical care in diabetes-2014. Diabetes Care 37(Suppl 1):S14-S80

6. Egede LE, Gebregziabher M, Echols C, Lynch CP (2014) Longitudinal effects of medication nonadherence on glycemic control. Ann Pharmacother 48:562-570

7. Lawrence DB, Ragucci KR, Long LB, Parris BS, Helfer LA (2006) Relationship of oral antihyperglycemic (sulfonylurea or metformin) medication adherence and hemoglobin A1c goal attainment for HMO patients enrolled in a diabetes disease management program. J Manag Care Pharm 12:466-471

8. Rolnick SJ, Pawloski PA, Hedblom BD, Asche SE, Bruzek RJ (2013) Patient characteristics associated with medication adherence. Clin Med Res 11:54-65

9. Rubin RR (2005) Adherence to pharmacologic therapy in patients with type 2 diabetes mellitus. Am J Med 118(Suppl 5A):27S-34S

10. Calip GS, Hubbard RA, Stergachis A, Malone KE, Gralow JR, Boudreau DM (2014) Adherence to oral diabetes medications and glycemic control during and following breast cancer treatment. Pharmacoepidemiol Drug Saf 24:75-85

11. van Herk-Sukel MP, van de Poll-Franse LV, Lemmens VE et al (2010) New opportunities for drug outcomes research in cancer patients: the linkage of the Eindhoven Cancer Registry and the PHARMO Record Linkage System. Eur J Cancer 46:395-404

12. World Health Organization. Collaborating Centre for Drug Statistics Methodology-ATC/DDD index. Available from www.whocc.no/atc ddd index. Accessed 15 Aug 2014

13. Catalan VS, LeLorier J (2000) Predictors of long-term persistence on statins in a subsidized clinical population. Value Health 3:417-426

14. Karve S, Cleves MA, Helm M, Hudson TJ, West DS, Martin BC (2008) An empirical basis for standardizing adherence measures derived from administrative claims data among diabetic patients. Med Care 46:1125-1133

15. Penfold RB, Zhang F (2013) Use of interrupted time series analysis in evaluating health care quality improvements. Acad Pediatr 13:S38 S44

16. Durbin J, Watson GS (1951) Testing for serial correlation in least squares regression. II. Biometrika 38:159-178

17. Karve S, Cleves MA, Helm M, Hudson TJ, West DS, Martin BC (2009) Good and poor adherence: optimal cut-point for adherence measures using administrative claims data. Curr Med Res Opin 25: 2303-2310 
18. Nicholas J, Charlton J, Dregan A, Gulliford MC (2013) Recent $\mathrm{HbA}_{1 \mathrm{c}}$ values and mortality risk in type 2 diabetes. Populationbased case-control study. PLoS One 8:e68008

19. Cohen HW, Shmukler C, Ullman R, Rivera CM, Walker EA (2010) Measurements of medication adherence in diabetic patients with poorly controlled $\mathrm{HbA}(1 \mathrm{c})$. Diabet Med 27:210-216

20. Comprehensive Cancer Centre the Netherlands (IKNL), Netherlands Cancer Registry, available from www.cijfersoverkanker.nl. Accessed 7 Jul 2014 [website in Dutch]

21. Sobin LH, Gospodarowicz MK, Wittekind C (2009) TNM classification of malignant tumours, 7th edn. Wiley, Oxford

22. Peyrot M, McMurry JF Jr, Kruger DF (1999) A biopsychosocial model of glycemic control in diabetes: stress, coping and regimen adherence. J Health Soc Behav 40:141-158

23. Zullig LL, Shaw RJ, Crowley MJ et al (2013) Association between perceived life chaos and medication adherence in a postmyocardial infarction population. Circ Cardiovasc Qual Outcomes 6:619625

24. Vigneri P, Frasca F, Sciacca L, Pandini G, Vigneri R (2009) Diabetes and cancer. Endocr Relat Cancer 16:1103-1123

25. Glucophage (metformin hydrochloride) [package insert] (2009) Princeton, NJ: Bristol-Myers Squibb

26. Misbin RI (2004) The phantom of lactic acidosis due to metformin in patients with diabetes. Diabetes Care 27:1791-1793

27. Lopez JM, Annunziata K, Bailey RA, Rupnow MF, Morisky DE (2014) Impact of hypoglycemia on patients with type 2 diabetes mellitus and their quality of life, work productivity, and medication adherence. Patient Prefer Adherence 8:683692

28. Curkendall SM, Thomas N, Bell KF, Juneau PL, Weiss AJ (2013) Predictors of medication adherence in patients with type 2 diabetes mellitus. Curr Med Res Opin 29:1275-1286 